\title{
WHR is a Better Predictor of Type 2 DM Among Urban Adults in Jos, North Central, Nigeria
}

\author{
${ }^{* 1}$ Aniekwensi Ezechukwu C and Fabian H Puepet ${ }^{2}$ \\ ${ }^{*}$ Consultant physician/Endocrinologist, Department of Internal medicine, Federal Medical Centre, Asaba, Nigeria \\ ${ }^{2}$ Professor of Medicine,Consultant Physician/Endocrinologist, Department of Internal Medicine, University of Jos/Jos University Teaching Hospital, \\ Jos, Nigeria
}

*Correspondence to: Dr Aniekwensi Ezechukwu, Federal medical centre, Asaba PMB 3001, Asaba, Delta State, Nigeria

Received: June 06, 2017; Accepted: June 21, 2017; Published: June 26, 2017;

\begin{abstract}
Background

Obesity is considered the strongest risk factor for Type $2 \mathrm{DM}$ and numerous studies have demonstrated this association.

Objective

To compare the Body mass index (BMI), the Waist Circumference (WC) and the Waist Hip-Ratio (WHR) in predicting Type 2 Diabetes.
\end{abstract}

Methods

This is a cross sectional study involving 709 subjects recruited from a multi-stage sampling method in the study location. Demographic data and anthropometric variables were obtained according to a standard protocol. Plasma glucose analysis in the fasting state and 2 hours after ingestion of $75 \mathrm{~g}$ glucose were done. We estimated the predictive value of obesity parameters WC, WHR, BMI in the prediction of diabetes. Receiver- Operator Characteristic (ROC) curves were used to determine the predictive power of each variable.

Results

The age range off our subjects was 20-89. There were 308 males (43.4\%) and 401 females (56.6\%). We documented 29 subjects with incident diabetes (previously undiagnosed) comprising 14 males and 15 females. The ROC curve showed that WHR had the highest AUC.

\section{Conclusion}

The ROC curve analysis indicated that WHR was better than WC and BMI in predicting type 2 diabetes.

\section{Introduction}

Obesity is an important risk factor for cardio metabolic diseases, including diabetes, hypertension, dyslipidaemia and coronary heart disease (CHD). Obesity is considered the strongest risk factor for type 2 diabetes [1]. Several leading health institutions including the WHO and the National Institute of Health (U.S.A) have provided guidelines for classifying weight status based on BMI $[2,3]$. These have agreed that men and women who have a BMI $\geq 30 \mathrm{~kg} / \mathrm{m}^{2}$ are considered obese and are generally at higher risk for adverse health events than are those who are considered overweight (BMI between 25.0 and 29.9kg/ $\mathrm{m}^{2}$ ) or lean (BMI between 18.5 and $24.9 \mathrm{~kg} / \mathrm{m}^{2}$ ). Therefore, BMI has become the gold "standard" for identifying patients at increased risk for adiposity - related adverse health outcomes [4].

Body fat distribution is also an important risk factor for obesityrelated diseases. Excess abdominal fat (also known as central or upper body fat) is associated with an increased risk of cardiometabolic disease.
Waist Circumference (WC) is often used as a surrogate marker of abdominal fat mass, because WC correlates with abdominal fat mass (subcutaneous and intra-abdominal) [5] and with cardiometabolic disease risk [6]. Men and Women who have waist circumference greater than $102 \mathrm{~cm}$ and $88 \mathrm{~cm}$, respectively are considered to be at increased risk for cardiometabolic disease [7].

IDF recently proposed new waist circumference cut off points as criteria for central obesity $[8,9]$. The values are ethnic and gender specific. European values are $94 \mathrm{~cm}$ for men and $80 \mathrm{~cm}$ for women, for Asians $90 \mathrm{~cm}$ for men and $80 \mathrm{~cm}$ for women. Sub-Saharan Africans are to use European values until more specific data are available.

\section{Methods}

We analysed the data of a population based cross-sectional study of 800 urban adults in jos, North Cental, Nigeria. In brief, a multistage sampling technique was used to select 800 subjects who met the inclusion criteria.In the first stage, Jos Municipality was selected , and in second stage, 2 wards were selected randomly from 40 wards by simple balloting and then in third stage a household survey was done and 800 subjects from 340 households selected systematically(every 
second household) were identified and invited to participate in the study.

The study procedure was explained to all subjects and written informed consent was obtained from each subject. A questionnaire (Appendix C) was administered to obtain relevant demographic, social and medical history. The questionnaire for data collection, physical measurements and biochemical parameters was adapted and modified from WHO STEPS instrument [10]. All anthropometric measurements were done standardized. The following anthropometric measurements were taken:

i. Weight $(\mathrm{kg})$ - Was measured to the nearest $0.1 \mathrm{~kg}$ with subjects in minimal clothing and without shoes using a standard weighing scale.

ii. Height $(\mathrm{m})$ - Was measured to the nearest $0.1 \mathrm{~cm}$ with a stadiometer with subjects barefoot and without headgear.

iii. Body Mass Index (BMI) - Was calculated as weight in $\mathrm{kg}$ divided by the square of height in meters $\left(\mathrm{m}^{2}\right)$ i.e. $\mathrm{kg} / \mathrm{m}^{2}$.

iv. Waist circumference $(\mathrm{cm})$ - Was measured to the nearest 0.1 $\mathrm{cm}$ using a non-stretch metric tape as the horizontal level at the mid-point between the lowest rib and the iliac crest.

v. Hip circumference $(\mathrm{cm})$ - Was measured to the nearest $0.1 \mathrm{~cm}$ as the largest circumference of the gluteal region or as the maximal circumference around the buttocks (posteriorly) and the pubic symphysis (anteriorly).

vi. Waist - to - Hip Ratio (WHR) - Was calculated as the waist circumference $(\mathrm{cm})$ divided by the hip circumference $(\mathrm{cm})$.

Fasting blood sugar was measured after 8-12 hours of overnight fast in the morning by the glucose oxidase method. Diabetes was diagnosed as Fasting plasma glucose $\geq 7.0 \mathrm{mmol} / \mathrm{l}$ and/or 2 hours plasma glucose $\geq 11.1 \mathrm{mmol} / \mathrm{l}$ after a $75 \mathrm{~g}$ oral anhydrous glucose load.

The WHO criteria for Obesity with BMI was used. BMI of 18.524.9 for normal, 25.0-29.9 overweight and $\geq 30.0$ as obese. Abnormal WC was done with IDF criteria of less than in males and less than in females. Abnormal WHR was determined using......

Statistical analyses was done with SPSS version 16 . The Receiver Operator Characteristic (ROC) curve analysis was used to determine which of the anthropometric indices best correlates with glucose intolerance.

\section{Results}

\section{Age and Sex distribution of Subjects}

Eight hundred subjects were enrolled into the study, of whom 709 responded and participated in the survey giving a response rate of 88.6\%. A total of 308 (43.4\%) male subjects and 401 (56.6\%) female subjects participated in the study giving a male to female ratio of 1 : 1.3. The mean (SD) age of study subjects was 43.21 (14.73) years with a range of 20 to 89 years. The mean (SD) age of the male subjects was 42.19 (15.3) years while that of female subjects was 43.99 (14.39) years, females being slightly older, however this difference was not significant, $(\mathrm{t}=1.62, \mathrm{p}=0.10)$. The age and sex distribution of the study subjects is shown in Table 1.

Table 1: Age and Sex distribution of Study Participants (Responders).

\begin{tabular}{|c|c|c|c|c|}
\hline \multirow{2}{*}{$\begin{array}{c}\text { Age } \\
\text { (Years) }\end{array}$} & \multicolumn{2}{|c|}{ Sex } & \multirow{2}{*}{ Total } & Percentage (\%) \\
\cline { 2 - 3 } & Males & Females & & \\
\hline $20-29$ & 76 & 77 & 153 & 21.6 \\
$30-39$ & 78 & 91 & 169 & 23.8 \\
$40-49$ & 50 & 90 & 140 & 19.8 \\
$50-59$ & 59 & 61 & 120 & 16.9 \\
$60-69$ & 29 & 54 & 83 & 11.7 \\
$\geq 70$ & 16 & 28 & 44 & 6.2 \\
\hline Total & $\mathbf{3 0 8 ( 4 3 . 4 \% )}$ & $\mathbf{4 0 1 ( 5 6 . 6 \% )}$ & $\mathbf{7 0 9}(\mathbf{1 0 0} \%)$ & $\mathbf{1 0 0}$ \\
\hline
\end{tabular}

$\mathrm{X}^{2}=11.26, \mathrm{P}=0.046$

The comparative ability of the Indices to correctly identify Diabetes mellitus was tested using the Receiver Operator Characteristic (ROC) Curve analysis.

\section{The ROC Curves for DM in Male subjects}

The ROC Curves of the Anthropometric Indices associated with the presence of DM in Male subjects are shown in Figure 8. The AUC's for BMI, WC, WHR were 0.79( $\mathrm{p}<0.001), 0.83(\mathrm{p}<0.001)$, $0.87(\mathrm{p}<0.001)$ respectively showing that although all the studied indices had significant abilities to identify DM in male subjects, WHR was the best in this population.

All the indices had significant AUC compared to the Null hypothesis true area of 0.5 . Since WHR had the highest AUC (0.87), as seen in the curves, it has the best predictive ability for DM in males.

\section{Discussion}

The ROC curve analysis for type 2 diabetes in men in this study had AUC's of $0.79,0.83,0.87$ for BMI, WC, WHR respectively while that of women in this study had AUC's of 0.74, 0.69, 0.74 for BMI, WC, WHR respectively.

The findings of this study showed that all the studied anthropometric Indices had significant ability to identify subjects with $\mathrm{DM}$, however WHR was superior to both $\mathrm{WC}$ and $\mathrm{BMI}$ in predicting Diabetes in men. In women, the WHR yielded equal predictive ability with BMI and both were superior to WC in predicting diabetes in women.

This contrasts with Caucasian studies $[11,12]$ where WC performed better than WHR as a predictor of type $2 \mathrm{DM}$. The ROC curve analysis for type 2 diabetes in men in this study had AUC's of 0.79, 0.83, 0.87 for BMI, WC, WHR respectively. These AUC's are higher than the ones obtained in the EPIC-Potsdam study [13] for type 2 diabetes in men with AUC's of 0.75, 0.76, 0.74 for BMI, WC, WHR respectively. For men, WHR had the highest AUC in this study while WC had the highest AUC in the EPIC-Potsdam study. In women, for type 2 diabetes, the AUC's in this study were $0.74,0.69,0.74$ which was lower than the AUC's in the EPIC-Potsdam study, 0.80, 0.83, 0.81 for BMI, WC and WHR respectively. BMI and WHR had the highest AUC in this study while WC had the highest AUC in the EPIC-Potsdam study.

In the D.E.S.I.R. study, [14] for type 2 diabetes, in men, BMI had a higher AUC than WC or WHR (0.77 as against 0.74 and 0.66$)$ while 
in women, WC had a higher AUC than BMI or WHR (0.82 as against 0.77 and 0.77$)$. This differs also from this study which in men, WHR had a higher AUC than BMI or WC (0.87 as against 0.83 and 0.79$)$ and in women, BMI and WHR had higher AUC's than WC (0.74 as against 0.69) [Figure 1].

\section{ROC Curve for DM in Males}
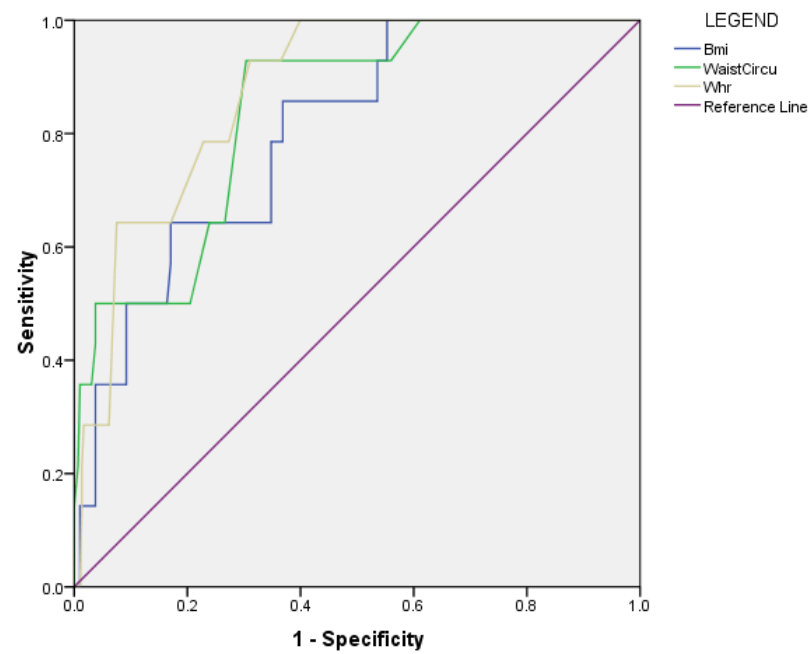

Figure 1: ROC Curves of the anthropometric Indices associated with the presence of DM in male subjects.

Thus while WC co-relates better and appears to be more sensitive than BMI or WHR in identifying type 2 diabetes in Caucasians, WHR co-relates better and probably more sensitive than BMI or WC in identifying type 2 diabetes in our study population. We observed that in our subjects while some had normal BMI and WC, such subjects may have abnormal WHR. This is reflected in the fact that Obesity was commonest using WHR in our study population. This was in agreement with the general observation that native Africans tend to have smaller physical frame than their Caucasian counterpart. This finding suggests that native Africans deposit fat differently from Caucasians whom appear to have larger waist circumference than Africans. Hoffman et al, found that Caucasians had a greater visceral adipose tissue mass and smaller subcutaneous adipose tissue mass compared with African-americans respectively [15]. Mechanisms to explain the racial difference in visceral adipose tissue content are lacking [15]. BMI performed least in its discriminative ability to identify DM in men but performed better than WC in women. BMI is unable to differentiate between fat mass and muscle mass and does not identify abdominal obesity which has been shown to correlate more strongly with obesity related health risks than peripheral obesity [16]. Some Nigerian studies also showed that BMI compared to other anthropometric indices performed poorly as an index of obesity among Nigerian adults with diabetes mellitus [17]. In our subjects with DM, body fat distribution represented by WHR was more sensitive than WC or BMI in identifying them. WHR may be a better measure of central obesity than WC in this population.

\section{Conclusion}

The results of our study contrasts with earlier studies in Caucasians with WHR predicting diabetes better than BMI and
WC and also discriminated diabetic from nondiabetic subjects with higher accuracy in men. Since obesity, metabolic syndrome, diabetes and cardiovascular diseases are highly prevalent in our population, this results emphasize the application of WHR as an appropriate discriminative tool for identification of diabetes.

\section{References}

1. WHO Expert Committee on Diabetes Mellitus: second report (1980). World Health Organ Tech Rep Ser 646: 1-80. [crossref]

2. World Health Organization (1998) Obesity: Preventing and managing the Global Epidemic: Report of a WHO consultation on Obesity. Geneva, WHO.

3. National Institute of Health (1998): National Heart, Lung and Blood Institute. Clinical guidelines on the identification, evaluation, and treatment of overweight and obesity in adults - the evidence report. Obes Res 6 (Supp1.2): S51-S209.

4. Klein S, Allison D, Heymsfield SB, Kelley DE, et al. (2007) Waist circumference and cardiometabolic risk. A consensus statement from shaping America's Health: Association for Weight Management and Obesity Prevention: NAASO, the Obesity Society, the American Society for Nutrition; and the American Diabetes Association. Diabetes Care 30: 1647-1652.

5. Pouliot MC, Depes JP, Lemieux S (1994) Waist circumference and abdominal saggital diameter: Best simple anthropometric indices of abdominal visceral adipose tissue accumulation and related cardiovascular risk in men and women. $A M$ J Cardiol 73: 460-468.

6. Kissebah AH, Vydelingum N, Murray R, Evans DJ, Hartz AJ, et al. (1982) Relation of body fat distribution to metabolic complications of obesity. $J$ Clin Endocrinol Metab 54: 254-260. [crossref]

7. Wang Y Rimm EB, Stampfer MJ, Willett WC, Hu FB (2005) Comparison of abdominal adiposity and overall obesity in predicting risk of type 2 diabetes among men. Am J Clin Nutr 81: 555-563. [crossref]

8. Alberti KG, Zimmet P, Shaw J; IDF Epidemiology Task Force Consensus Group (2005) The metabolic syndrome--a new worldwide definition. Lancet 366: 1059 1062. [crossref]

9. Alberti KG, Zimmet P, Shaw J (2006) Metabolic Syndrome-a new worldwide definition. A Consensus Statement from the International Diabetes Federation. Diabet Med 3: 469-480.

10. World Health Organisation (2008) WHO STEPwise approach to chronic disease risk factor surveillance- Instrument version 2.0, Questionnaire file. Department of Chronic Diseases and Health promotion.

11. Wei M, Gaskill SP, Haffner SM, Stern MP (1997) Waist circumference as the best predictor of Noninsulin dependent diabetes mellitus (NIDDM) compared to body mass index, waist hip ratio and other anthropometric measurements in Mexican Americans- a 7-year prospective study. Obes Res 5: 16-23.

12. Falkenberg M (2001) Check your waist circumference! Overweight, obesity and abdominal obesity risk factors of type 2 diabetes. Lakartidningen 98: 3520-3522.

13. Schuleze SB, Heidemann C, Schienkiewitz A, Bergmann MM, Hoffmann K, Boeing $\mathrm{H}$ (2006) Comparison of Anthropometric characteristics in predicting the incidence of Type 2 Diabetes in the EPIC - Potsdam study. Diabetes care 29: 1921-1923.

14. Balkau B, Sapinho D, Petrella A, Mhamdi L, Cailleau M, Arondel D, et al. (2006) and the D.E.S.I.R. Study group: Prescreening tools for diabetes and obesityassociated dyslipidemia: Comparing BMI, Waist and WHR. The D.E.S.I.R Study. Eur J Clin Nutri 60: 295-304.

15. Hoffman DJ, Wang Z, Gallagher D, Heymsfield SB (2005) Comparison of visceral adipose tissue mass in adult African Americans and whites. Obes Res 13: 66-74. [crossref]

16. Kahn SE, Prigeon RL, Schwartz RS, Fujimoto WY, Knopp RH, et al. (2001) Obesity, body fat distribution, insulin sensitivity and Islet beta-cell function as explanations for metabolic diversity. J Nutr 131: 354S-60S. [crossref]

17. Okubadejo N, Fasanmade A (2004) Concomitant hypertension and type 2 diabetes mellitus in Nigerians: Prevalence of obesity and its indices compared to normotensive diabetics. Niger Med J 45: 79-83.

Citation:

Aniekwensi Ezechukwu C and Fabian H Puepet (2017) WHR is a Better Predictor Of Type 2 DM Among Urban Adults in Jos, North Central, Nigeria. Endocrinol Diabetes Metab J Volume 1(2): 1-3 